\title{
Non-linear acoustic imaging of ice properties
}

\author{
J. Y. Guigné, G. B. Crocker and P. Hunt \\ Centre for Cold Ocean Resources Engineering, Memorial University of Newfoundland, St. Fohn's, \\ Newfoundland A1B 3X5, Canada
}

\begin{abstract}
Non-linear acoustic techniques have been used to investigate the properties of a pond-ice cover. The field tests showed that the position of the lower and upper surfaces, as well as some internal bubble layers, could be located with centimetre-scale resolution. These results are presented, along with an outline of parametric array theory and a description of the instrumentation. The data indicate that dynamic modelling of the acoustic response of ice will enable remote estimation of some of the bulk physical properties of the ice such as thickness, roughness and possibly brine volume. It follows that this approach to non-destructive imaging of ice properties may find application in a wide range of ice-engineering problems.
\end{abstract}

\section{INTRODUCTION}

An understanding of the interactions between floating ice and engineered structures is essential to the economic and environmentally sound development of the Earth's polar regions. Governments and industry are looking increasingly toward these regions, and the polar oceans in particular, as areas for future development. This increased activity has brought with it an increased demand for detailed information on the properties of ice which control its behaviour as a material. These physical properties include its thickness, temperature, brine volume, crystal structure and related mechanical properties such as Young's modulus, Poisson's ratio, compressive strength, tensile strength and shear strength. Significant advances in our understanding of ice properties and ice-structure interaction have beerı achieved in the past two decades; however, much of the work to date has focused on the large and meso-scale properties of sea and lake ice, or small-scale properties of artificially grown ice. Although measurements at these scales are suitable for many purposes, numerous applications require systems capable of non-destructive sampling of natural ice masses with millimetre-scale resolution.

For example, the small-scale underside roughness of sea-ice floes is important in boundary-layer studies, the entrapment of oil under ice and in determining the overall drag coefficient of the floe. There is also a need for more information on the spatial and temporal variability of ice properties, as it is often the extreme rather than the average properties which control the forces exerted on a structure (see Sanderson, 1988).

Although non-destructive (remote) techniques are unlikely to provide direct measurements of the mechanical properties mentioned above, a system capable of measur- ing fundamental physical properties would be extremely valuable, as the mechanical properties could be inferred from it. Several attempts at remotely imaging these basic ice characteristics have been made, employing a variety of techniques including $\mathrm{HF}$ radar and electromagnetic induction. These techniques vary widely in terms of range, resolution and degree of signal processing and interpretation required. Although some of these systems have been successfully used to measure (or infer) ice thickness in specific circumstances, they are incapable of clearly imaging both the upper and lower ice surfaces, and do not show any promise for extension to the measurements or estimation of other fundamental properties. Linear acoustic techniques (Jezek and others, 1990) have been used to infer ice type on the basis of reflectioncoefficient probability-density functions. While they suggest that the intensity of the reflected energy may be influenced by internal structure (such as bubbles) near to the ice/water interface, they could not identify individual structures or quantify the contribution of the features to back-scattering. This system is also limited in its ability to resolve the air/ice interface. Rajan and others (1990) made acoustic cross-hole tomographic sections of lake and bay ice showing the shear and compressional wave-speed profiles at 30 and $90 \mathrm{kHz}$. This system does provide information which can be correlated to ice properties but requires that the sensors be moved vertically through holes in the ice sheet. An acoustic approach which may overcome many of these limitations is nonlinear acoustics.

The advent of non-linear acoustic technology, specifically the parametric array, allows a broad-band source to be realized in practice (Berktay and others, 1979; Kon$\mathrm{rad}, 1979)$. The non-linear interaction of sound in water was first described by Westervelt (1963). If two intense 
sound beams are coaxially transmitted into the water, due to the non-linearity of the medium new secondary frequencies are produced, including sums and differences of the primary propagation frequencies. This creates a parametric array. At the low-difference frequencies, a very narrow beam is produced with minimal or no sidelobe energy. The band width of these frequencies is large, approximately that of the primaries. This is of special importance to the sounding of ice since the length of an acoustic pulse, which is determined by the signal's band width, places limits on the minimum thickness of ice and the thickness of the internal layers that can be unambiguously resolved. It is well documented and understood in geophysics that, if an acoustic pulse length is of the same dimension as the thickness of the medium being sensed, the resulting returns from the top and bottom of the layer will interact causing interference (Guigné and others, 1990). The two time events which delineate the boundaries of the layer would then become blurred or incoherent.

A short pulse with a narrow beam, as provided by the parametric array through the non-linear process, also permits stronger signal-to-reverberation ratios to be achieved when scattering by air bubbles in the ice is dominant or present (Guigné and Chin, 1989a). Scattering by small voids within the ice (bubbles, brine pockets, brine drainage channels and cracks) severely restricts acoustic returns from conventional sources. It is rare that coherent short impulses of the order obtainable with parametric arrays can be achieved with current commercial echo sounders. This makes non-linear acoustics a particularly attractive approach.

Wallerstedt (1989) specifically verified the use of a non-linear parametric sonar to measure sea-ice thickness. It was observed that coherent parametric reflected pulses off both lower (ice/water) and upper (ice/air) surfaces were received. In this paper, we present the results of an experiment aimed at determining the feasibility of using non-linear acoustic techniques to image remotely ice properties. The field trials were conducted on Long Pond, Newfoundland, in March 1990. At this time, the pond surface was covered by a fairly uniform fresh-water ice cover approximately $0.32 \mathrm{~m}$ thick, and completely free of snow. The water depth at the measurement site was approximately $2.6 \mathrm{~m}$.

\section{INSTRUMENTATION AND TECHNIQUES}

An efficient technique for generating a parameteric array is to employ a process called self-demodulation (Berktay, 1965). If a modulated carrier frequency signal is radiated from the acoustic transducer, non-linear interaction takes place in the water between all frequency components present. The secondary signal produced in the water contains a band of different frequencies and has a time-domain form proportional to the second-time derivatives of the square of the modulation envelope. The secondary pressure produced by a parametric array may be expressed as

$$
p(\vec{r}, t)=D \frac{\partial^{2}}{\partial t^{2}} p_{\mathrm{m}}^{2}(t)^{*} i(\vec{r}, t)
$$

where $i(\vec{r}, t)$ is the impulse response of the parametric ar- ray at position $\vec{r},{ }^{*}$ is the convolution and $D$ is a constant which is a function of the frequency-independent parameters (Ceen and Pace, 1981; Pace and Ceen, 1983). For under-ice imaging, the axial wave form of the parametric array is normally incident on the ice/water interface in the near field of the carrier frequency. In this case, the impulse response of the axis can be assumed to take the form of a delta function. The primary frequency modulation is $p_{\mathrm{m}}(t)$ and may take many forms, depending on the application. For a broad-band secondary signal, a form for $p_{\mathrm{m}}(t)$ which is easily implemented is the cosine bell

$$
p_{\mathrm{m}}(t)=\frac{1}{2}\left(1+\cos 2 \pi f_{\mathrm{c}} t\right)\left[U\left(t+\frac{1}{2 f_{\mathrm{c}}}\right)-U\left(t-\frac{1}{2 f_{\mathrm{c}}}\right)\right]
$$

where $U($.$) is the unit step function and f_{\mathrm{c}}$ is the carrier frequency. The normalized on-axis secondary wave form resulting from this modulation is

$$
\begin{aligned}
p(t)=\frac{1}{2} & \left(\cos 2 \pi f_{\mathrm{c}} t+\cos 4 \pi f_{\mathrm{c}} t\right)\left[U\left(t+\frac{1}{2 f_{\mathrm{c}}}\right)\right. \\
& \left.-U\left(t-\frac{1}{2 f_{\mathrm{c}}}\right)\right] .
\end{aligned}
$$

This wave form has a normalized spectrum

$$
P_{0}(f)=\frac{\pi f}{2 f_{\mathrm{c}}}\left[\frac{\sin A}{A} \frac{1}{\left(2+f / f_{\mathrm{c}}\right)}+\frac{\sin B}{B} \frac{1}{\left(1+f / f_{\mathrm{c}}\right)}\right]
$$

where $A=\left(2-f / f_{\mathrm{c}}\right) \pi$ and $B=\left(1-f / f_{\mathrm{c}}\right) \pi$. This spectrum peaks at $f=1.5 f_{\mathrm{c}}$. Figure 1 shows a "raised cosine bell" modulated carrier and the self-demodulation signal which would be received on a broad-band hydrophone located on the acoustic axis of the transmitting transducer. The pressure spectrum of the secondary or demodulated signal is also shown in this figure (Guigné and others, 1989).

The acoustic system used in this experiment was developed at C-CORE, Memorial University (Guigné and Chin, 1989b). The probe consisted of a broad-band, narrow beam source with centre frequencies from 60 to $120 \mathrm{kHz}$. Signal acquisition and processing was performed with a digital data-acquisition system. The transducer and hydrophone were mounted on a rotating arm suspended $0.65 \mathrm{~m}$ below the ice/water interface (Fig. 2). The support frame was designed such that the rotating arm could be locked in any one of eight evenly spaced positions.

\section{ICE CONDITIONS}

The experiment was conducted on 6 March 1990. At this time the ice cover on Long Pond was completely free of snow and had a fairly smooth, uniform upper surface giving the impression of lateral homogeneity. Before the acoustic imaging was performed, an ice block was removed for analyses of crystal structure, density and bubble distributions. The hole left by the removal of the block served as the centre point for the sensor mount. The thickness of the block varied between 


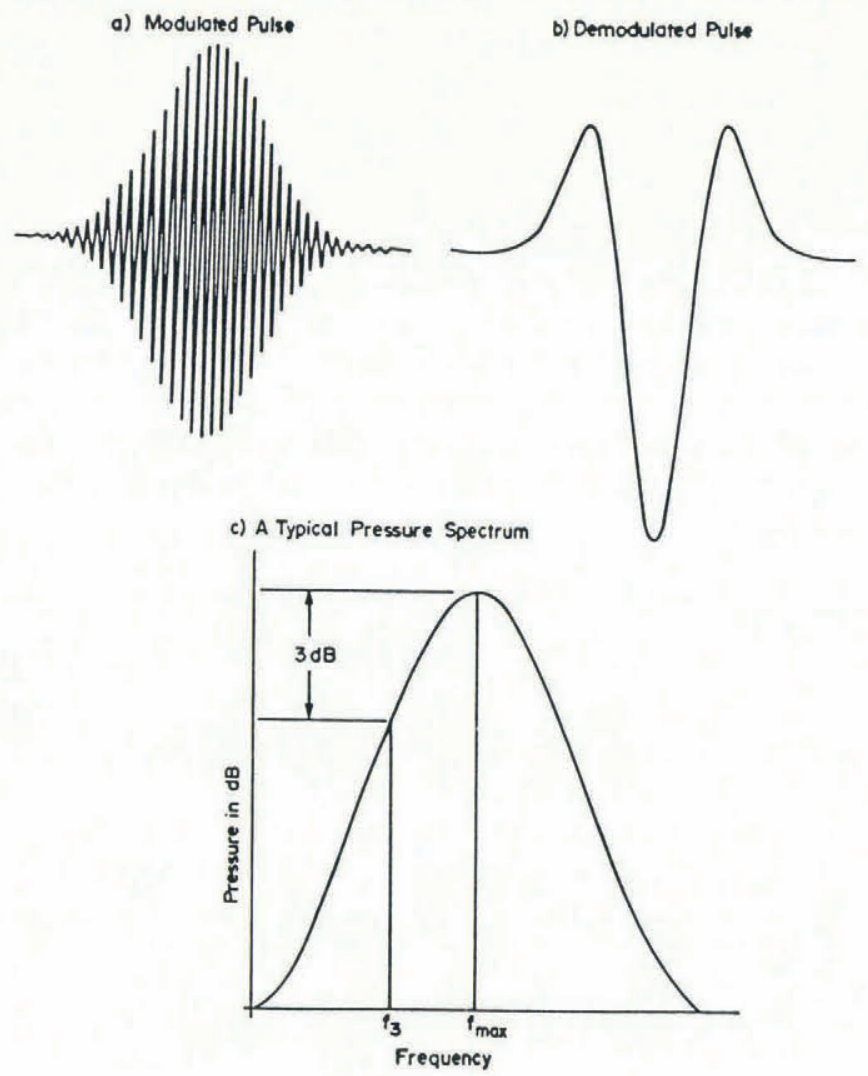

Fig. 1. The raised cosine bell modulated carrier waveform (a), self-demodulation signal which would be received on a broad-band hydrophone (b) and the pressure spectrum of the demodulated signal (c). (After Guigné and others, 1989.)

0.31 and $0.33 \mathrm{~m}$, and its density was estimated to be $880 \mathrm{~kg} \mathrm{~m}^{-3}$, or about $4 \%$ air space by volume. The density estimate was obtained by cutting a part of the block into a cuboid shape, measuring its volume and weighing it on an electronic balance.

Of greater importance to acoustic imaging was the vertical distribution of bubbles in the ice sheet. The bubbles ranged in shape from nearly perfect spheres to thin tubes arranged with their long axes vertical (Fig. 3 ). Vertical thin sections were prepared on a microtome and photographed between crossed polaroids. This procedure revealed that the ice was composed of long columnar crystals $0.02-0.03 \mathrm{~m}$ in diameter and $0.10-0.15 \mathrm{~m}$ in length. There were no apparent associations between the bubble layers and the crystal structure. The absence of smaller crystals and/or a polycrystalline layer at the surface of the ice suggests that the ice formed in the initial stages of growth had melted and run off. The complex bubble stratigraphy was the result of extremes of temperature and precipitaiton during the growth history. The 1990 ice season was characterized by cycles of cold $\left(-10^{\circ}\right.$ to $-20^{\circ} \mathrm{C}$ ) windy weather followed immediately by warm temperatures $\left(5^{\circ}\right.$ to $\left.10^{\circ} \mathrm{C}\right)$ and heavy rain. This resulted in rapid changes in the rate of growth at the ice/water interface, and produced the strongly stratified, bubbly ice sheet shown in Figure 3. The bubble patterns are primarily a function of the ice-growth rate and amount of dis-

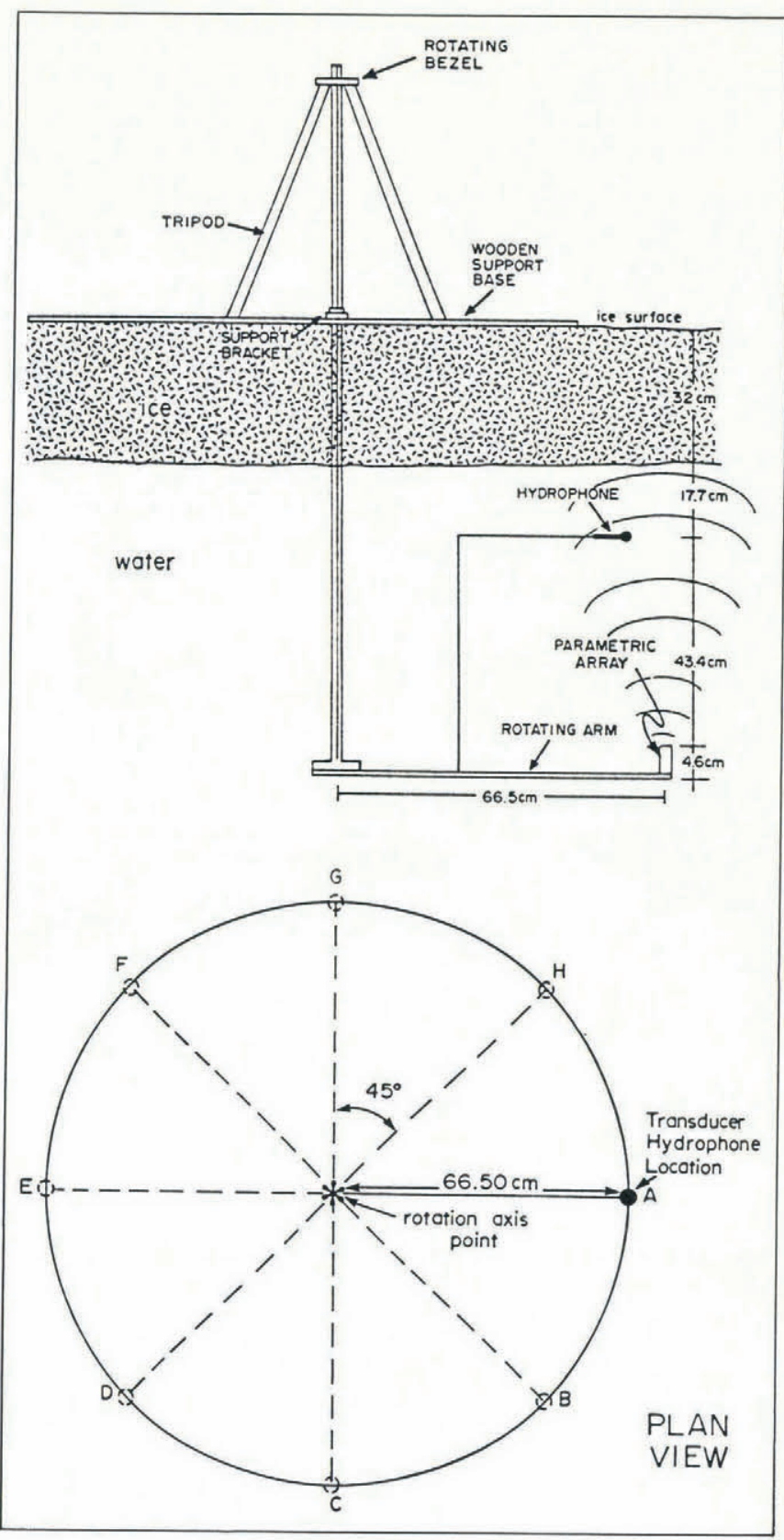

Fig. 2. Schematic representation of the instrument set up on Long Pond, 6 March 1990.

solved air in the water (Hobbs, 1974). In general, large bubbles are produced during periods of slow growth, and small bubbles are the result of rapid growth. The shape of the bubbles is a function of the supply of dissolved air, and to a lesser extent thermal metamorphosis after initial bubble formation (Maeno, 1967). When the air supply is constant, the bubbles tend to become cylindrical, while a discontinuous air supply results in spherical shapes. In the case of a shallow pond, the bubble densities and shapes may also be influenced by gaseous releases from the sediments.

Ice temperatures were not measured; however, in the absence of a snow cover, it can be assumed that the icesurface temperature was close to the ambient air temperature during the measurement period $\left(\approx-9^{\circ} \mathrm{C}\right)$. It can also be assumed that the ice at the lower interface was at the freezing temperature $\left(0^{\circ} \mathrm{C}\right)$. 


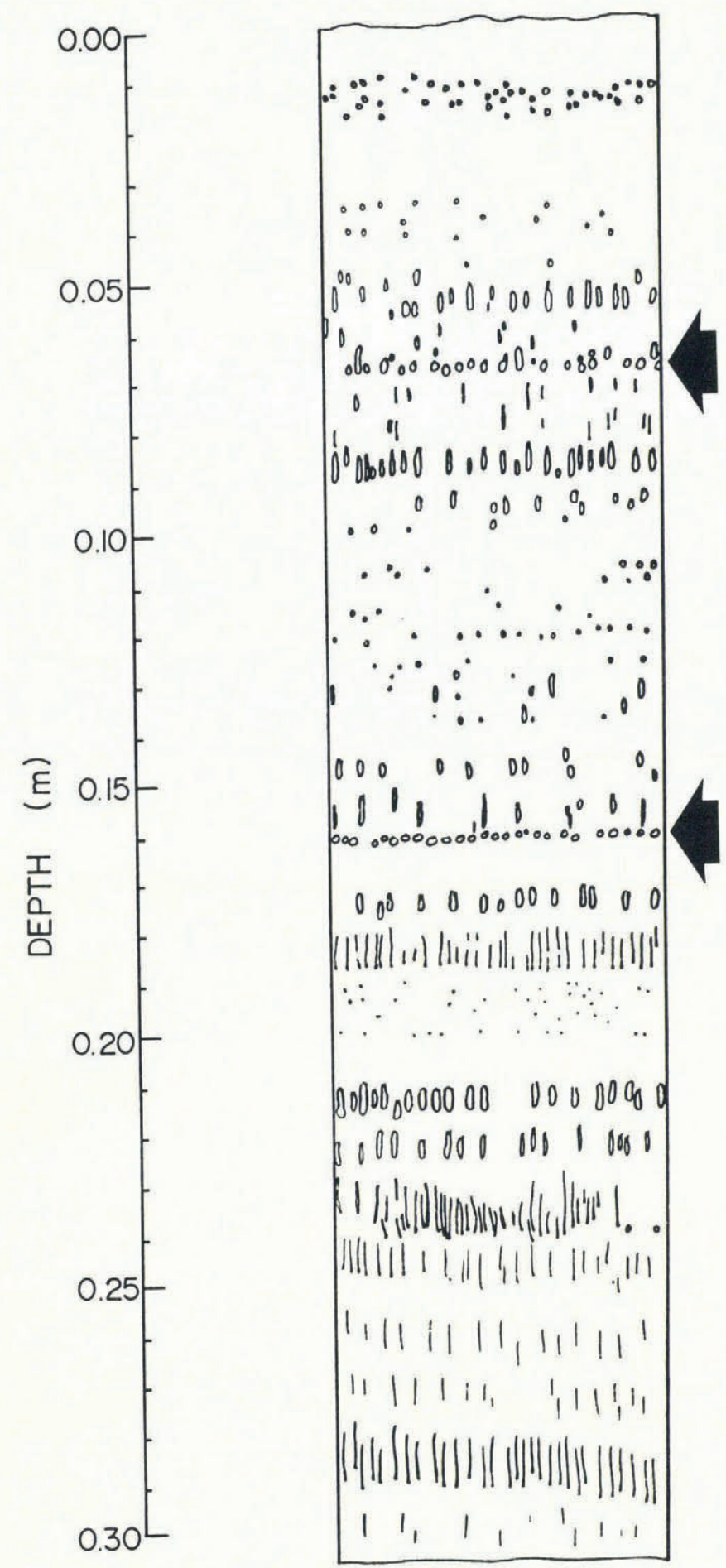

Fig. 3. Sketch of the bubble structure found in the ice block. A depth of $0.0 \mathrm{~m}$ represents the ice/air interface, and the two arrows indicate the depths of two internal reflecting surfaces $R_{2}$ and $R_{3}$ (see text).

\section{DATA ANALYSIS}

In order to measure accurately the position of the ice/water interface, it is necessary to measure accurately the time interval between the outgoing pulse and the returned echo. The conventional use of the maximum positive amplitude as a time reference involves the identification of a specific peak on the time trace. This approach is sensitive to phase changes which may occur upon re- flection, to ambient noise, and to variations in rate of rise time, which are controlled by the water/ice impedance and ice properties. These variables affect the sign and amplitude of the real valued time signal. Uncertainties can therefore arise in defining a representative maximum amplitude point.

To enhance and mathematically better describe the signal content, its real-time history can be restated as an analytic signal using the Hilbert transform (Guigné, 1986; Guigné and Chin, 1989a). To obtain the envelope signal, the time-domain signal $s(t)$ is made complex. By applying the Hilbert transform, an analytic signal $z(t)$ is obtained:

$$
\begin{gathered}
z(t)=s(t)+j \tilde{\mathrm{s}}(t) \\
z(t)=E(t) \exp (j \phi(t)) \\
=\|z(t)\| \exp (j \phi(t))
\end{gathered}
$$

or

where $\tilde{\mathbf{s}}(t)$ is the Hilbert transform of the time signal $s(t), E(t)$ is the energy envelope signal or magnitude of $z(t), \phi(t)$ is the instantaneous phase signal and $j$ is $(-1)^{\frac{1}{2}}$ (Bendat, 1975).

Although most time signals contain rapidly oscillating components, these oscillations are removed by the Hilbert transform during the formation of the envelope. This allows for a more complete and detailed study of the signal, since the imaginary part is included in the calculations. The Hilbert transform offers an elegant model for ice-structure mapping since ambiguities in referencing signal peaks are greatly reduced (by an order of magnitude). When a complex signal is used, the resulting envelope is a positive function which resembles an energy-time curve, hence the need to be concerned with polarity does not exist. What emerges is a clearer description of the actual energy parcel, and the influences on it during travel through the ice. This permits the travelling energy to be represented graphically on a logarithmic scale, thus providing a wide dynamic display range.

Furthermore, the velocity computed from the Hilbert transform time traces is a group velocity; the velocity of the maximum energy of the wave-train pulse. Differences between the group velocity and the phase velocity (the wave speeds of the different frequencies within the pulse) are influenced by the attenuation occurring within the ice. The Hilbert transform provides a good mathematical basis for modelling the group velocity.

\section{RESULTS}

Figure 4 shows a single acoustic record collected at transducer position A. The top part of the figure shows the real part of the time history and the lower part shows the instantaneous amplitude of the same record. The instantaneous amplitude represents a pseudo-energy envelope. The significant events labelled on Figure 4 are synthesized into the following points:

(i) The event labelled D represents the direct arrival from the transducer. The transducer/hydrophone separation of $0.434 \mathrm{~m}$ and the arrival time of $306 \mu \mathrm{s}$ yield a velocity of sound in water of $1418 \mathrm{~m} \mathrm{~s}^{-1}$. 

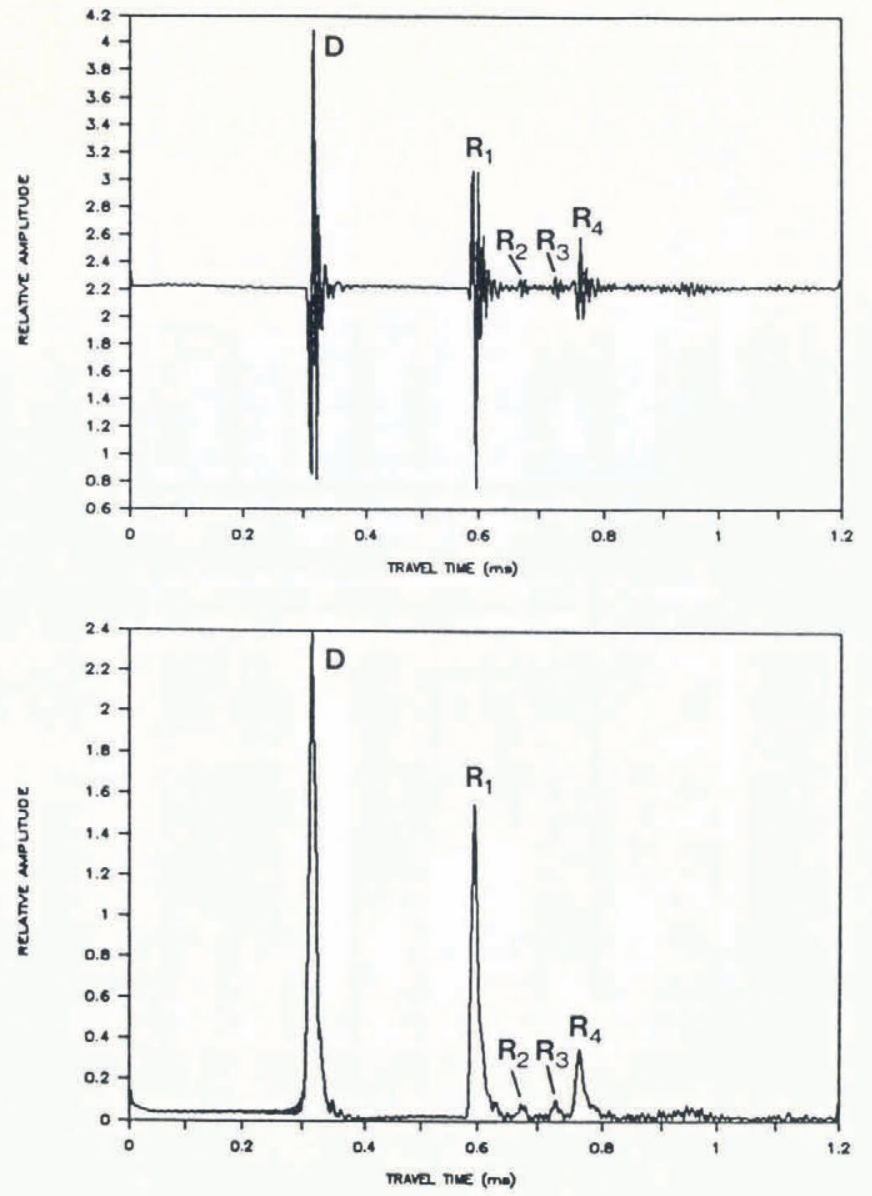

Fig. 4. An acoustic record collected at location $A$ in Figure 2. The diagram at the top shows the real-time history. The lower diagram shows the instantaneous amplitude of the same record.

(ii) The event labelled $R_{1}$ represents the first reflected energy from the ice/water interface.

(iii) The events labelled $R_{2}$ and $R_{3}$ represent internal reflections from within the ice.

(iv) The event labelled $\mathrm{R}_{4}$ represents the reflection from the ice/air interface. The polarity reversal between $R_{1}$ and $R_{4}$ results from the low acoustic impedance of the air above the ice, in comparison with the acoustic impedance of the ice itself. The average one-way travel time through the ice of $90 \mu \mathrm{s}$, along with the average ice thickness of $0.32 \mathrm{~m}$, gives an average acoustic velocity in the ice of $3550 \mathrm{~m} \mathrm{~s}^{-1}$.

Figure 5 shows a part of the eight acoustic records. The top part shows the real-time histories and the bottom part shows the instantaneous amplitudes. The acoustic data have an automatic gain correction applied to enhance the weaker internal reflections. The following can be noted from an examination of these records:

(i) The ice-bottom reflection varies with spatial position, revealing the roughness of the underside of the ice. The position of the $R_{1}$ reflection varies by a maximum of $22 \mu$ s of two-way travel time, corresponding to a thickness variation of $0.016 \mathrm{~m}$.
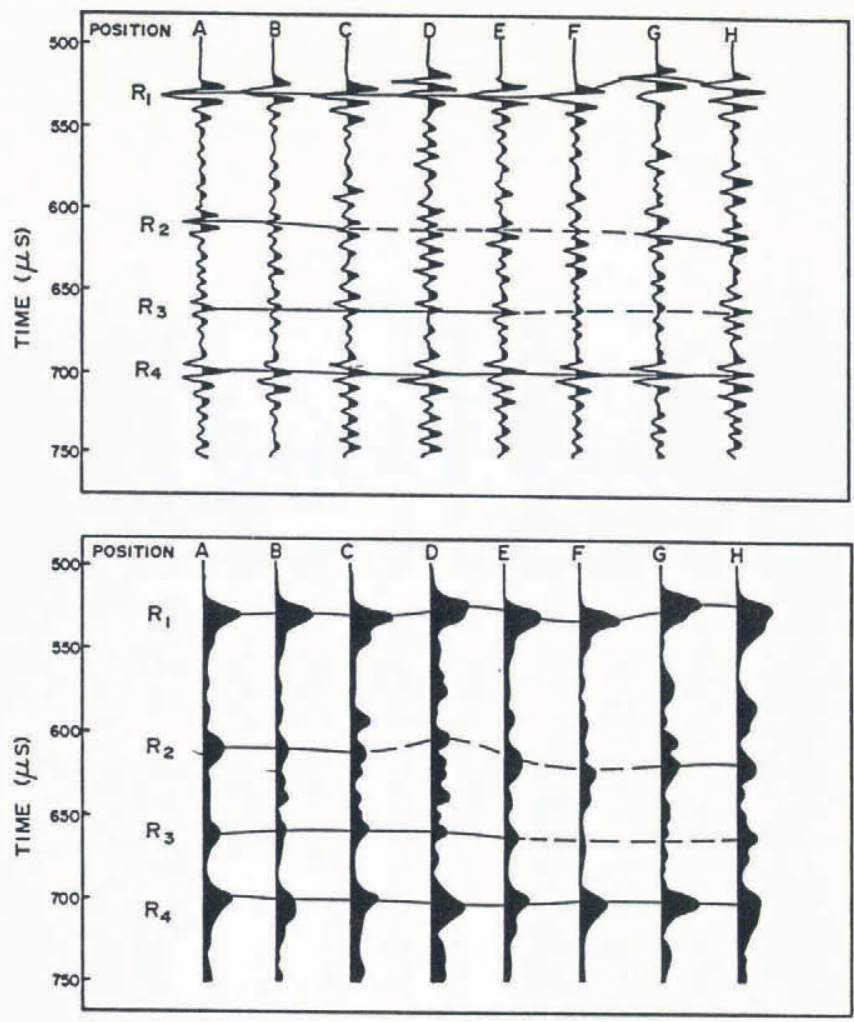

Fig. 5. Real-time histories of the eight acoustic records, with automatic gain control (upper), and instantaneous amplitudes with automatic gain control (lower). The position letters refer to Figure 2.

(ii) The character of the $R_{1}$ reflection also varies between positions. Interference of two or more returns is evident at positions C, D, G and H. This is indicative of reflections from closely spaced reflectors near the ice/water interface.

(iii) The $R_{2}$ reflection is seen to be present across most of the acoustic records. This event is marked with a solid line except at positions D, E and F, where its location is not clearly evident. The one-way travel time from the $R_{1}$ to the $R_{2}$ reflector is $40 \mu \mathrm{s}$, placing $\mathrm{R}_{2} 0.14 \mathrm{~m}$ from the ice/water interface, assuming constant velocity.

(iv) The $R_{3}$ reflection can also be followed across the section except at $\mathrm{F}$ and $\mathrm{G}$ where it is denoted by a dashed line. The one-way travel time between $\mathrm{R}_{1}$ and $R_{3}$ is $65 \mu \mathrm{s}$, placing this reflector $0.23 \mathrm{~m}$ above the ice/water interface.

(v) The $R_{4}$ reflection is marked with a solid line at all positions. The strength of the reflection and its reversed polarity clearly indicate that this is the ice/air interface.

Figure 6 shows the magnitude of the $R_{1}$ reflection as a function of position for the three different acoustic pulse frequencies. The large magnitude of the $60 \mathrm{kHz}$ pulse at position $\mathrm{B}$ is questionable and may have resulted from an incorrect gain recording during data collection. The difference in magnitude of the $R_{1}$ reflection at positions 

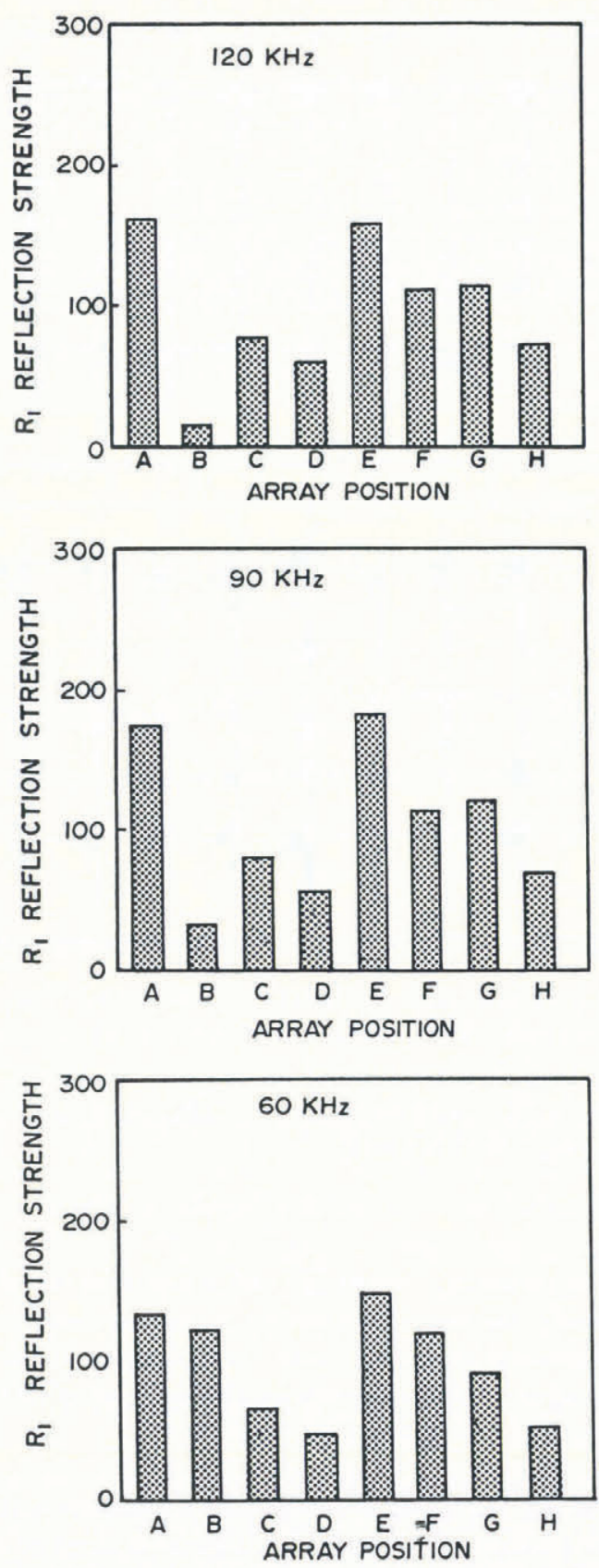

Fig. 6. The magnitude of the $R_{1}$ reflection as a function of position for the three different acoustic pulse frequencies.

A through $\mathrm{H}$ is indicative of spatially varying physical properties (such as density) of the ice/water interface.

The percentage of the energy which was transmitted through the ice and returned to the hydrophone as a function of array position is shown in Figure 7 for the three different frequency acoustic pulses. The largest percentage energy return occurred at position $\mathrm{F}$. It is noteworthy that internal reflections were not well pronounced at this position. The amount of energy transmitted provides an indication of the presence of scatterers in the ice. The redistribution of energy by these scatterers can be used to characterize the nature of the ice matrix.

It is interesting to see that the two internal reflections correspond to the only two layers composed pre-
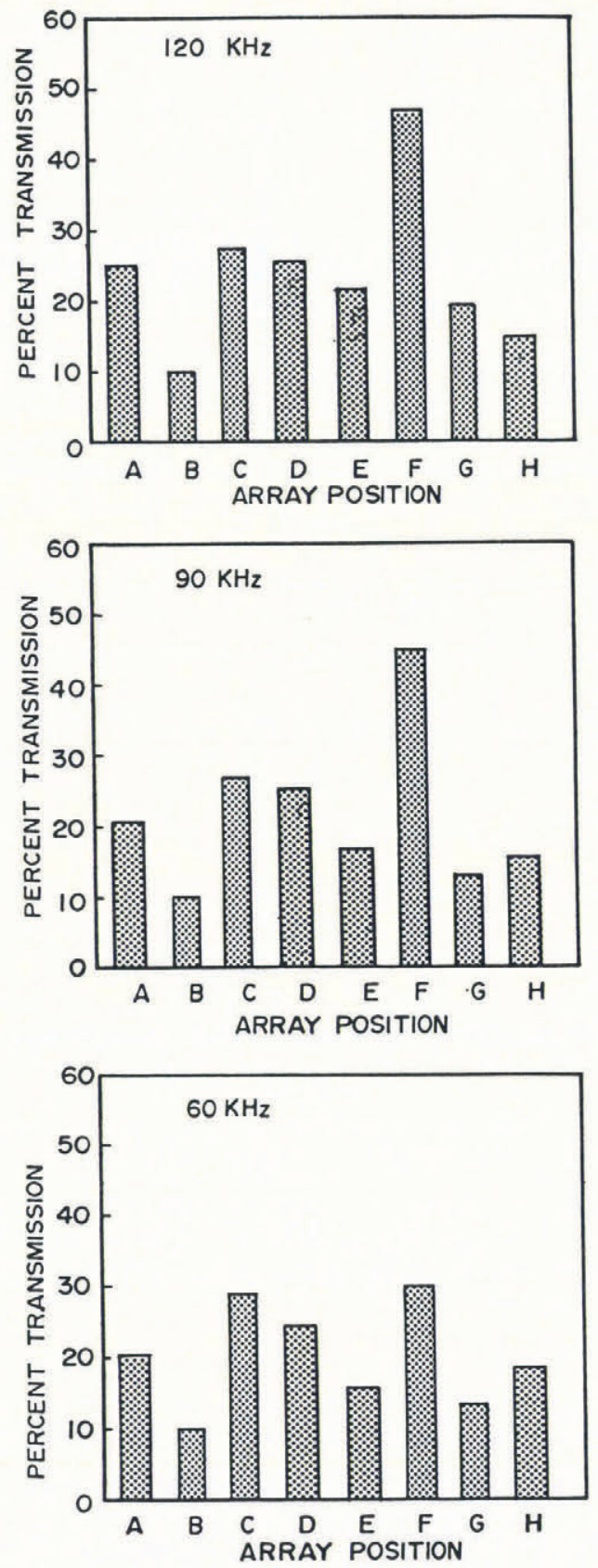

Fig. 7. The percentage of energy transmitted through the ice and returned to the hydrophone.

dominantly of spherical bubbles (refer to Figure 3). This is somewhat misleading since it is not the shape of the bubbles that is of the greatest importance, but rather the bubble volume in the plane perpendicular to the direction of acoustic energy flow. These two layers stand out because they project low-density planes to the acoustic signal.

\section{CONCLUSIONS}

The results described above are extremely encouraging, and were obtained without matching the properties of the acoustic signal to the acoustic properties of the ice for optimal performance. Nevertheless, in its present configuration, the equipment has been shown to produce infor- 
mation on both the bulk properties of the ice cover and its internal structure. These results suggest a wide range of applications.

The internal structure of ice, in particular bubble and brine volumes, is crucial to the mechanical behaviour of the material. In fact, most engineering properties of sea ice can be empirically linked to this single parameter. The underside roughness is another important property which can be measured with precision through the use of non-linear acoustics. The capability for detection of both the ice/water and ice/air interfaces also facilitates measurements of surface roughness. The advantages of detecting both interfaces for ice-thickness measurements are obvious. The narrow beam width, omission of side lobes and precise coherent timing of the pulse return enable these measurements to be made with far greater accuracy than is possible using conventional systems. The approach also tends to prevent the generation of transverse Rayleigh waves within the ice, thereby minimizing volume reverberation effects. The reduction of multipath propagation and possible shear-wave effects concurs with the research findings of Wallerstedt (1989).

It is likely that this technique can also be applied to a number of other ice-related measurement problems. Since boundaries between materials with different properties can be resolved so clearly, measurements of slush or water layers within, or on top of an ice sheet are also possible. Similarly, the thickness and perhaps the porosity of sub-ice platelet and frazil-ice accumulations can be investigated. The latter in particular is of considerable practical importance to the hydraulics of ice-covered rivers and lakes.

There are also several devel, ;ing fields of ice research where accurate measurement of ice thickness is essential. Wadhams (1990) has suggested that the impact of $\mathrm{CO}_{2}$-induced global warming can already be seen in thickness changes of the Arctic pack-ice cover. More accurate thickness data, fully exploiting the spatial and temporal resolution obtainable with the parametric array, would greatly improve our capability for using the sea-ice cover as an indicator of climatic change. Similarly, high-resolution underside roughness profiles would provide insight into the fractal nature of ice at much smaller scales than is currently possible (Bishop and Chellis, 1989; Connors and others, 1990). While our experiment, which has dealt with thin fresh-water ice, has not proven the feasibility of these applications, it does show the great potential of non-linear acoustics to ice-measurement problems.

This study has shown that parametric array technology can be used to measure the thickness of thin floating ice sheets, and identify internal layering with centimetre accuracy. In addition to detecting the presence and thickness of these different layers, the acoustic returns infer something about the bulk properties of the material. The acoustic energy balance within the ice is controlled by attenuation, reflection and scattering, which are all in turn controlled by the physical composition of the ice. Guigné and others (1989) have shown how analyses of the shape of the spectral distortion can be used to garner information about attenuation, which can be linked to physical parameters such as density and porosity. These dynamic models were successfully ap- plied to the more complex case of sea-bed soils (Guigné and others, 1989), and similar techniques should be applicable to floating ice masses. Measurements of brine volume and crystal structure are particularly promising.

The non-linear acoustic approach has two additional attributes which make it extremely attractive. The first is that sampling is non-destructive. This permits repetitive mapping of ice features, and the development of a time series of ice properties. The second is its ability to make large numbers of measurements in short periods of time. This facilitates measurements of the spatial variations in ice properties. Spatial information is important in all areas of ice physics and ice engineering, but so far it has been largely neglected because of the difficulties associated with making the large number of measurements required. Programs to develop many of these ideas, and produce a prototype system of hardware and software tailored specifically to ice-engineering problems, are already in place.

\section{ACKNOWLEDGEMENTS}

Field support was provided by D. Christian of C-CORE. The constructive comments of two anonymous reviews resulted in significant improvements to this paper, and are greatly appreciated.

\section{REFERENCES}

Bendat, J. 1975. The Hilbert Transform and applications to correlation measurements. Brüel and Kjar Monograph.

Berktay, H. 1965. Possible exploitation of non-linear acoustics in underwater transmitting applications. $J$. Sound Vib., 2(4), 435-461.

Berktay, H., B. Smith, H. Braithwaite and M. Whitehouse. 1979. Sub-bottom profilers with parametric sources. In Underwater Applications on Nonlinear Acoustics. Session 1.2. Bath, England, Bath University. Institute of Acoustics, 1-10.

Bishop, G. and S. Chellis. 1989. Fractal dimension: a descriptor of ice keel surface roughness. Geophys. Res. Lett., 16(9), 1007-1010.

Ceen, R. and N. Pace. 1981. Time domain study of the terminated parametric array. In Advances in Underwater Acoustics. Session 6. Bath, England, University of Bath. Institute of Acoustics, 1-10.

Connors, D., E. Levine and R. Shell. 1990. A smallscale under-ice morphology study in the High Arctic. CRREL Monogr. 90-1, 145-151.

Guigné, J. 1986. The concept design and experimental evaluation of an acoustic sub-seabed interrogator. (Ph.D. thesis, University of Bath.)

Guigné, J. and V. Chin. 1989a. Acoustic imaging of an inhomogeneous sediment matrix. Mar. Geophys. Res., $11,301-317$.

Guigné, J. and V. Chin. 1989b. Optimization of terminated parametric arrays for underwater use. Proceedings of the Annual Meeting of the Canadian Acoustical Society, Halifax, Nova Scotia, October $16-19,1989,35-38$.

Guigné, J., G. Nicholas and V. Chin. 1989. Dynamic 
extraction of sediment attenuation from subbottom acoustic data. J. Geophys. Res., 94(B5), 5745-5755.

Guigné, J., N. Rukavina, P. Hunt and J. Ford. 1990. An acoustic parametric array for measuring the thickness and stratigraphy of contaminated sediments. J. Great Lakes Res., 17(1), 120-131.

Hobbs, P. 1974. Ice physics. Oxford, Clarendon Press.

Jezek, K., T. Stanton, A. Gow and M. Lange. 1990. Influence of environmental conditions on acoustical properties of sea ice. J. Acoust. Soc. Am., 88(4), 19031912.

Konrad, W. 1979. Applications of the parametric array source. In Underwater Applications on Non-linear Acoustics. Session 1.1. Bath, England, Bath University. Institute of Acoustics, 1-9.

Maeno, N. 1967. Air bubble formation in ice crystals. In Oura, H., ed. Physics of Snow and Ice. International Conference on Low Temperature Science, ... 1966 ... . Proceedings, Vol. 1, Pt. 1. Sapporo, Hokkaido University. Institute of Low Temperature Science, 207-218.
Pace, N. and R. Ceen. 1983. Time domain study of the terminated parametric array. J. Acoust. Soc. Am., 73(6), 1972.

Rajan, S., J. Doutt and G. Frisk. 1990. Determination of compressional and shear wave speed profiles and attenuation in sea ice. J. Acoust. Soc. Am., 87, Supplement 1 .

Sanderson, T. 1988. Ice mechanics; risks to offshore structures. London, etc., Graham and Trotman Inc.

Wadhams, P. 1990. Evidence for thinning of the Arctic ice cover north of Greenland. Nature, 345(6278), 795-797.

Wallerstedt, R. 1989. Review of parametric ice thickness sonar testing at Resolute Bay, Canada - 1987. CRREL Special Report, 89-39, 427-450.

Westervelt, P. 1963. Parametric acoustic array. J. Acoust. Soc. Am., 35, 535-537.

The accuracy of references in the text and in this list is the responsibility of the authors, to whom queries should be addressed.

MS received 3 October 1990 and in revised form 15 July 1991 